\title{
What Should an Ideal Emergency Psychiatry Service be Like?
}

\author{
SteVen WhITE, Wellcome Trust Research Fellow, Institute of Psychiatry, De Crespigny Park, London SE5 8AF
}

At a time of financial stringency, it may seem perverse to speculate about the ideal. Nevertheless, recent experience of working in the psychiatry emergency room (ER) of a large teaching hospital in Texas set me thinking about the requirements for a really satisfactory emergency psychiatry service (EPS); one which would run smoothly and be acceptable to the clients, their families and the agencies involved in making referrals; one the staff running it would feel comfortable with and get satisfaction from working in; one which would provide a comprehensive and really adequate service, rather than just a finger in the dike.

What is the role of an EPS?

The prime function is to provide specialist advice on the evaluation and management of acute disturbances of mental state and behaviour. The initial step is to decide whether a given situation actually constitutes an emergency. Presenting problems will not always require or be amenable to intervention emergently, but may be dealt with most appropriately by re-direction to non-emergency follow-up. This may be in an out-patient clinic or through social work or counselling agencies.

If a true emergency does exist, the next question is whether the disordered mental state is the result of organic disease. This, of course, is a particularly important step in the evaluation. If physical disorders are missed at this stage, much of the subsequent intervention may be inappropriate and, at worse, harmful or hazardous. An open mind must be kept on the presence of organic disease. The possibility should be considered again as the evaluation continues and more information becomes available. If such disease does exist, its nature will determine the course to be taken. This may be complete transfer of care to an appropriate medical or surgical team. Alternatively, it may involve consultation and joint management with other physicians. This may be necessary where the patient's behaviour is so disordered as to make management in the standard way in a general medical environment difficult or impossible.

If an emergency does exist and there is no evidence of organic disease, the next decision to be made is whether there is a mental illness. This will, again, have a major influence on the direction of the subsequent management.

Psychiatrists are commonly asked, especially in emergencies, to give an opinion on whether a particular individual is dangerous, either to himself or to other people. This actually represents an extraordinarily difficult task. It involves an estimate of probabilities on the basis of specific information about the patient, together with an understanding of features of the past history and current mental state which may be predictive of dangerousness. The reliability of such judgements will inevitably be rather limited, no matter how much information is available, since people may always behave unexpectedly. Nevertheless, the emergency psychiatrist must attempt some assessment of dangerousness and, indeed, this role figures prominently in the operation of the Mental Health Act.

Finally, a balanced judgement must be made as to the safest and most helpful course of action to be taken. This must take into account the needs and responsibilities of the patient himself, his family and other agencies involved, and society at large. It must not lead to any undue or unwarranted restrictions being placed on the patient; nor should it result in unreasonable demands or hazards for his family and others around him.

Having summarised very briefly the elements of emergency intervention in psychiatry, what facilities are required to achieve a high standard of care?

\section{The setting}

Nobody likes sitting waiting to be seen in a casualty department or clinic. However, an adequate waiting area is particularly important in emergency psychiatry. A proportion of those waiting will be in acute emotional distress and may be accompanied by several supporting family members. Some will be restless and want to wander about. Some will be intoxicated with alcohol or other drugs and be drowsy or anxious and excitable. Others will be suspicious, fearful, withdrawn or irritable. A few will have the potential for sudden eruptions of seriously disturbed behaviour. This is a difficult and sometimes dangerous mixture. What is required is a waiting area with plenty of space and seating arranged so that people do not have to be closer to each other than they want. It needs to have some degree of privacy and to be away from the mainstream of activity. At the same time, it must be organised so that the staff are able to maintain a general surveillance of what is going on.

There should be a room to which seriously disturbed, agitated or intoxicated patients can be taken immediately on arrival. This is the psychiatric equivalent of the medical resuscitation room and should be able to fulfil the same function. That is, it should provide an environment suitable for the rapid evaluation of dangerously unstable situations, with facilities available to bring events back under control. In a psychiatric setting, this means a place where patients 
can be examined rapidly for the presence of physical disease or injury, and where those whose behaviour is disturbed or hazardous can be quickly and effectively medicated if this becomes necessary. Space should be available for this to be done in privacy, away from the general waiting area. Just as with a medical resuscitation room, this area should be kept ready and available for immediate use. There should be an additional room equipped for routine physical examinations and for the drawing of blood samples.

Sufficient interview rooms should be available to provide a setting for assessment in reasonable comfort. They should be large enough to enable family interviews involving more than one professional worker on occasion. Whilst privacy is essential, isolation is inadvisable. The interview rooms should be arranged so that assistance can be summoned rapidly if it is needed to deal with a dangerous or violent situation.

A recurrent practical problem in emergency psychiatry is the disposition of patients whom it is felt cannot safely be sent home immediately, but who may avoid hospital admission if they can be observed under suitable conditions for a further period of 12-48 hours. Examples include:

(1) Drug intoxications or withdrawal states of mild-tomoderate severity, not appearing to need full general medical care, but requiring observation in case this becomes necessary later and to ensure the safety of the patient and others.

(2) Patients who are felt to be '?organic', to allow time for further observation, investigation and consultation with colleagues.

(3) Those who are considered to be at risk for suicide or violent behaviour but where this seems likely to be a transient hazard related to drugs or alcohol, or some acute psychosocial stressor, so that discharge within $\mathbf{4 8}$ hours is a reasonable prospect.

(4) 'Acute situational disturbances' in which it seems that a person will be able to return home once a sudden dislocation of his social relationships has been remedied.

(5) Patients whose mental state or behaviour in the emergency room has made them inaccessible to satisfactory evaluation at that time.

There are various solutions to this problem. One general hospital I have worked in had a small ward adjacent to the casualty department, staffed by SRNs and covered by the medical house officers. This could keep overnight patients with mild DTs, other drug intoxication and withdrawal states, or drug overdoses not requiring general medical admission. These patients were observed along with those with minor head or orthopaedic injuries. They were usually reviewed by psychiatrists the following morning and if they were not fit for discharge home by that time arrangements were made for their in-patient care elsewhere. Another hospital operates a similar system, allowing a temporary stay overnight in psychiatric beds, without this constituting a formal admission. This permits observation in conditions of safety with nursing and medical care available.

In Texas, the psychiatry ER itself contained a 'holding area' with rooms where patients could sleep, permitting observation and re-evaluation over a period of up to 24 hours. There was good continuity of care and someone first seen in the ER during one shift could often be reviewed by the same doctor the following morning. If discharge was still felt to be inappropriate, transfer would be arranged to the nearby mental diagnostic centre. This was a closed unit where patients could be further evaluated and treated for up to a week or so. They would then either be discharged to out-patient care or transferred to the State Hospital for a longer psychiatric admission.

\section{Immediately available facilities}

About $2 \%$ of the patients seen in the psychiatry ER in Texas had organic disease severe enough to require emergency admission to general medical or surgical beds. This percentage included patients previously assessed in the medical and surgical ERs as well as outside referrals and walk-ins. Organic disease must be sought vigorously if a small but constant proportion of patients is not to be managed inappropriately. "Cleared by medicine-psychiatry please see" should not allow the possibility of significant organic disease to be dropped altogether from consideration-all the more so since adequate physical examination of a patient whose mental state is disturbed in a busy casualty department may be extremely difficult to achieve.

It is important that the EPS should have free access to emergency laboratory facilities for routine blood tests. Availability of toxicology screening is essential, since drugrelated abnormalities of the mental state are so commonly encountered. Chest X-rays and ECGs should be obtainable if needed, particularly when elderly confused patients are seen for evaluation.

In most centres, emergency CT scanning is available only on request from an on-call neurologist or neurosurgeon. Certainly I do not know of a hospital where psychiatrists can themselves request an emergency scan directly. However, by whatever route, it is valuable for an EPS to have access to this facility if it is available. If done sensibly, this would be cost-effective and enable the rapid detection of patients whose mental symptoms are due to cerebral tumours, subdural haematoma and subarachnoid or intracerebral haemorrhage. The number of such patients will not be large, but does seem to be fairly constant. Rapid identification of these conditions clearly has a major impact on management.

Since neurological disease may quite often present with acute disturbances of the mental state it is important, in any case, that emergency psychiatrists should be able to get a neurological opinion. In Texas, consultation from an inhouse neurologist was available 24 hours a day. This was very helpful and provided a quick route to CT scanning. Availability of consultations from general physicians and surgeons is also important.

\section{The staff}

Psychiatrists at various levels of training will be required in sufficient force to maintain a satisfactory throughput of patients. In the United States, especially in large urban 
centres, attending psychiatrists may specialise in emergency psychiatry and work full-time in that setting. In Texas, for example, the Director of the Psychiatry ER was an Associate Professor in the medical school and spent a significant part of his time each day in the ER, seeing patients with residents and medical students. Other psychiatrists of attending status worked there on a sessional basis. As a result, for much of the day, doctors of consultant grade were actually in the ER, evaluating patients and supervising the junior staff. This is an excellent arrangement for patient care and for teaching.

Medical students took a full part in the activities of this department. They would interview patients and present them to the residents or attending psychiatrists, discussing their thoughts about diagnosis and management. They would then see the patient again together and decide what should be done. Another feature was the involvement of a range of professions in the running of the service. Clinical psychology interns, social work and pastoral counselling trainees would all spend time working in the ER. They would operate like the medical students, with all patients being reviewed at some stage by a physician. This system worked well. It greatly assisted the running of the department and enabled use to be made of special skills which these groups possessed in counselling, family work and crisis intervention.

Ideally, the nursing staff on an EPS would be doubly trained, with general and psychiatric nursing qualifications and practical experience in both areas. This is particularly important for an EPS in a general hospital setting, or anywhere where large numbers of patients with alcohol and drug-related problems or recent drug overdoses and confusional states are seen. These patients require both careful physical monitoring and specialist psychiatric care. Nursing staff could, too, be more directly involved in patient assessment than in traditionally the case.

Since much emergency psychiatry includes crisis intervention in situations arising from major psychosocial stresses, direct participation of social workers in the day-today running of an EPS is essential. It would, preferably, have its own full-time social workers, who would be involved in the evaluation of patients. They would, perhaps divide their time between the clinic itself and related work in the community. Specialist counsellors working in areas such as drug and alcohol abuse, rape crisis intervention or familiar with the cultural background and needs of particular ethnic groups are a valuable resource. Opinions should also be available from other psychiatrists with experience in specialised fields, such as child and adolescent psychiatry.

It can be diff:cult to keep track of all the facilities for the mentally ill available in a community. It is helpful to have someone who is an expert in this and who can facilitate liaison between the EPS and other agencies. In the Texas department, someone-usually a master's level clinical psychologist-was present in the ER at all times to advise and co-ordinate admissions to other hospitals, hostel placements and referrals to special support groups and community organisations. It is actually cost effective to employ such a person, since it maximises the full and co-ordinated use of the available resources.

Finally, a system for dealing effectively with episodes of seriously disturbed or violent behaviour must always be rapidly available if an EPS is to run safely. In the United States, hospitals employ security guards who assist in such incidents, which they do well. In this country, medical and nursing staff will usually be expected to deal with the situation directly themselves, sometimes with assistance from other hospital staff. An adequate number of trained staff must be immediately available to restrain physically a violent patient and give medication if this becomes necessary to bring the situation back under control. In extreme circumstances-such as the seriously disturbed person with a weapon which he has already used or will not surrenderrapid support from outside agencies, in this case the police, is needed. Effective lines of communication with these agencies and a pre-established working relationship with the EPS is important.

\section{Supporting resources}

So far, I have dealt only with the running of the EPS itself. What affiliated resources are necessary for a good service? First of all, a route for admission to specialist psychiatric beds is essential for those patients who are undoubtedly acutely mentally ill and requires immediate hospital care. As with the rest of emergency medicine, nothing brings a busy EPS to a standstill faster than difficulties in finding beds for patients who have already been evaluated and clearly need to be admitted. Ideally, these beds would be on the same site as the EPS itself. There should be close links between the psychiatrists running the EPS and the ward. Probably the best arrangement would be to have an acute admissions and treatment unit with its beds committed to patients from the EPS. One or more consultant psychiatrists with supporting junior medical staff would run the ward and the EPS, dividing their time between the two areas. Nursing, social work and other services would be arranged in the same way. This would provide an excellent system of care, it would make administrative sense and would be good for staff training and research.

Such a combined unit should have attached out-patient clinics, which would provide follow-up for patients who had been discharged from the ward or seen in the EPS but not admitted. Whatever the arrangement, there must be a system for the immediate follow-up of patients seen in an EPS. This may be very short-term, to deal with an acute crisis, to support the patient while he is waiting for an appointment in another clinic or to review progress following the starting of medication.

An active EPS will encounter a wide range of problems, touching most areas of psychiatry. It should, consequently, have access to specialist clinics which can provide evaluation and treatment in fields such as drug and alcohol abuse, psychogeriatrics, mental impairment, forensic psychiatry, eating disorders and psychosexual dysfunction. These need not necessarily all be in the same hospital but administrative links for referral should be well-established. 


\section{Comment}

I do not know of a department providing an EPS which fulfils all of these specifications for an ideal service. Nevertheless, some manage to offer something quite close to it, despite less than ideal circumstances. I do not think any of the things which I have suggested are unduly extravagant. Indeed, relative to the cost of providing adequate facilities for dealing with acute trauma or medical emergencies, the funding required for most of what $I$ have put for- ward is probably relatively modest. Part of the problem is, perhaps, this. The facilities needed for good acute medical care seem fairly clear-cut. There is little difficulty in justifying the provision of resuscitation rooms, ECG monitors, cardiac arrest teams and so on. In contrast, just what it is that is required to provide good emergency psychiatric care may be more elusive. I hope that I have been able to draw attention to some of the practical requirements for a satisfactory service.

\section{Patients at a Psychiatric Walk-in Clinic - Who, How, Why and When}

Camilla Haw, Senior Registrar, Regional Secure Unit, St Bernard's Hospital, Uxbridge Road, Southall, Middlesex, (formerly Registrar, Maudsley Hospital); Colin Lanceley, Senior Registrar, St Augustine's Hospital, Chartham, Canterbury, Kent (formerly locum Senior Registrar, Maudsley Hospital) and STEVEn Vickers, Research Assistant, Department of Computing, Imperial College, 180 Queen's Gate, London, SW7

In recent years there has been increasing emphasis on community-based psychiatric services. If these facilities are to be comprehensive they must include ways of assessing and managing crises and avoiding unnecessary hospitalisation. One way of meeting these needs is to provide small walk-in clinics in the community. A number of clinics have been established and more are planned. Although clients may refer themselves, access is usually limited to office hours and often only to a few hours each day.

In contrast to these small local facilities, there exist a few larger psychiatric walk-in clinics which operate a 24 hour service for all patients regardless of catchment area. One such unit is the Maudsley Emergency Clinic, though recently its 24 hour operation has been threatened by Health Service cuts. The organisation of the clinic and some of the demographic characteristics of its clientele have already been described. ${ }^{1,2,3}$

Our study aimed to answer a number of questions-is a 24 hour clinic an expensive luxury? When do people present to the clinic? Are they sicker at night? How far do people travel for help? Are clients good judges of their need for psychiatric help? Are GPS better judges? Do people abuse the clinic?

\section{The study}

All visits to the clinic during July and August 1985 were studied. For each visit the following information was derived from the clinic's log book and stored on computer: name, age, sex, date and time of arrival, referral source, whether the patient lived within the Maudsley's catchment area, distance from the Maudsley to the patient's home, diagnosis-derived from ICD-9 (where several diagnoses were given the one most relevant to the presenting problem was taken), management plan, e.g. admission, 'guesting' overnight for review the following morning, out-patient referral, follow-up appointment at the clinic, discharge. Where details were missing the case notes were studied. By arrangement, the Emergency Clinic also sees the psychiatric patients who present to King's College Casualty Department out of hours or who arrive in office hours but without a referral letter. These presentations to King's constituted $14 \%$ of the index visits during the study period.

Statistical tests. Proportions of populations were compared using the normal approximation to the binomial distribution where sample sizes were permitted, or in some cases using the binomial distribution directly.

Who uses the clinic? 650 patients made 1,050 visits during the study period. $56 \%$ were men. Mean age was 36.7 years (s.d. 14.3). Drunks were included and assumed to be suffering from alcoholism. They were not otherwise included in the analysis, except under the timing of visits. This is because it is the clinic's policy not to interview drunk patients and so no details, other than their name and arrival time, were recorded.

Schizophrenia was the most frequent diagnosis $(21 \%)$ and psychotic patients were commoner than neurotic ones $(36 \% \vee 23 \%)$. Neurotic and psychotic depression constituted $27 \%$ of cases. 